P0189

\title{
MODELING REFLECTION PROPERTIES OF ROAD SURFACES BY DATABASE METHOD
}

\author{
Wenyi Li et al.
}

DOI 10.25039/x46.2019.PO189

from

CIE x046:2019

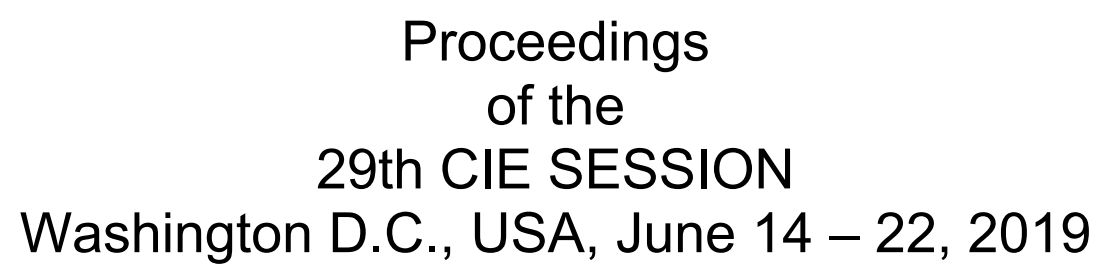

(DOI 10.25039/x46.2019)

The paper has been presented at the 29th CIE Session, Washington D.C., USA, June 14-22, 2019. It has not been peer-reviewed by CIE.

(C) CIE 2019

All rights reserved. Unless otherwise specified, no part of this publication may be reproduced or utilized in any form or by any means, electronic or mechanical, including photocopying and microfilm, without permission in writing from CIE Central Bureau at the address below. Any mention of organizations or products does not imply endorsement by the CIE.

This paper is made available open access for individual use. However, in all other cases all rights are reserved unless explicit permission is sought from and given by the CIE.

CIE Central Bureau

Babenbergerstrasse 9

A-1010 Vienna

Austria

Tel.: +4317143187

e-mail: ciecb@cie.co.at

www.cie.co.at 


\title{
MODELING REFLECTION PROPERTIES OF ROAD SURFACES BY DATABASE METHOD
}

\author{
Li, W. ${ }^{1}$, Zhang, Z. ${ }^{2,}{ }^{3}$, Yang, Y. ${ }^{2,}{ }^{3}$, Liu, M. ${ }^{1}$, Shen, H. ${ }^{1 *}$ \\ ${ }^{1}$ Fudan University, Shanghai, CHINA, ${ }^{2}$ China Road Transport Inspection and Certification Hi- \\ tech CO. Ltd., Beijing, CHINA, ${ }^{3}$ National Center for Quality Supervision \& Inspection of \\ Traffic Safety Facilities, Beijing, CHINA \\ * shenhaiping@fudan.edu.cn
}

DOI 10.25039/x46.2019.PO189

\begin{abstract}
This paper proposes a new method to model r-tables without an analytical expression, but by database method. A so-called 'knowledge base' for road surfaces is setup, which is constructed by verified r-tables. The data model is trained and built up by some selected r-tables, then predictions are made for the other r-tables in the knowledge base, and prediction errors are then analysed to verify the model. In this paper, 287 r-tables of dry road surfaces is tested. The model is built up by its $r$-tables with even number indices. The r-tables with odd number indices are then predicted. Results show that $90 \%$ of the prediction errors are below $15 \%$, and a great number of examples are distributed around $5 \%$. Only two worst cases are of error around $50 \%$. These results are much better than those we obtained by analytical expression methods. By this database model, if we have a large enough data base with reliable r-tables, covering road surfaces of different materials, service and weather conditions, we can make predictions for most road surfaces with a few measured value, then make a more precise road/street lighting design.
\end{abstract}

Keywords: Road lighting, Road surface, Reflection, R-table, Model

\section{Introduction}

For a precise designing of road/street lighting, the reflection properties (described by a reduced luminance coefficient table, short as r-table) of the road surface should be known. Since measurement of a full $r$-table is costly and time consuming, a better and practical way is to measure finite number of reduced luminance coefficients under several specified angles, then make a prediction for the full r-table by using a model. Extensively investigations have been carried out by scientists, trying to get an analytical expression model with an acceptable prediction precision:

Anisotropic Gaussian model, which was obtained from the theoretical formulation of Fresnel reflection (Ward, 1992).

Schreder model, which was proposed in (Debergh, 2008), and was related with Memphis and Coluroute gonio reflectometer. In this model, interpolation was used to obtain the whole r-table by a small number of measured reduced luminance coefficients at the intersection of the row corresponding to tan $y$ and of the column corresponding to $\beta$ as $r_{\mathrm{y} \beta}$.

Massart model, which works with the EP-diagram, uses an elliptic plus two nonlinear functions to describe the contour of an EP diagram containing the whole information of a r-table (Erbay, 1974).

$S_{1}$ and $S_{2}$ index matching model. We also proposed a relatively simple, but not an analytical expression model (Li, 2014). First we compute out the $S_{1}$ and $S_{2}$ values of a large number of road samples whose $r$-tables have been known, then for a sample to be predicted, the r-table in the database whose $S_{1}$ and $S_{2}$ values closest to those of this sample is chosen to be its $r$-table.

We also made a comparison study for all these four models. For the $287 \mathrm{r}$-tables we calculated, results showed that, the Anisotropic Gaussian model got an average overall error of $45.0 \%$, the model in Debergh and Embrechts's paper got an average overall error of $41.3 \%$, the Massart model's average overall error was $26.5 \%$. When using the $S_{1}$ and $S_{2}$ index matching model, the average overall error was $14.2 \%$. 
This paper proposes a new method to model r-tables without an analytical expression, but by a database method, to obtain higher model precision, even than the $S_{1}$ and $S_{2}$ index matching model.

\section{CIE r-table system}

The current system used for the calculation of road surface reflection properties and surface classification is defined in the CIE 66-1984 (CIE, 1984). The reflection properties of a road surface are described by a set of luminance coefficients which are dependent on the angles as illustrated by Figure 1. The angles are,

Y angle of incidence, the angle between the direction of incident light and the upward vertical;

$\alpha \quad$ angle of observation, the angle in a vertical plane, relative to the horizontal, at which the light is transmitted from the reflection point $P$ to the observer's eyes;

$\beta \quad$ angle of intersection, the angle in between of the vertical plane of incident light and the vertical plane of observation, with considers point $P$ as the intersection point; the angle between the vertical plane of observation and the road axis. Since most road surfaces are almost completely isotropic, the influence of $\delta$ can be neglected.

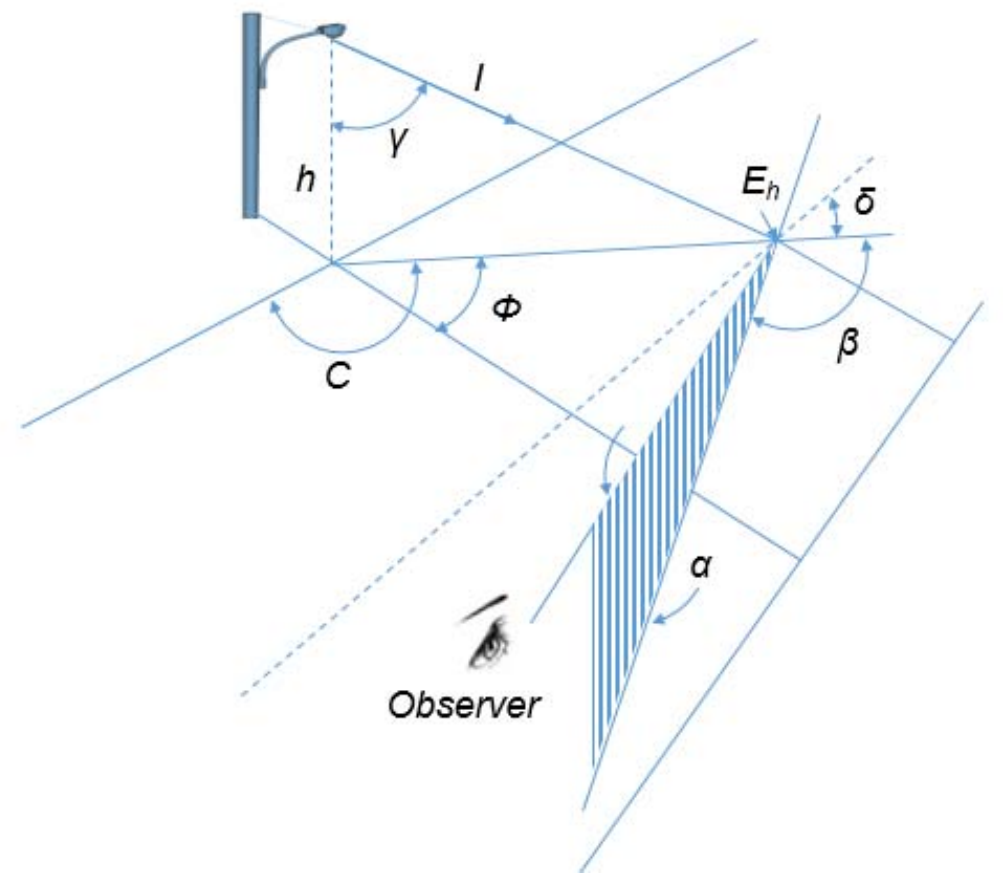

Figure 1 - Illustrator of angles upon which luminance coefficient $(q)$ is defined

The luminance coefficient $q$ is the ratio of the luminance on the measured road surface to the illuminance on the same measured area for the given angles of observation and incident light. Thus

$$
L_{p}=E_{H} \cdot q(\alpha, \beta, \gamma)=\frac{I(c, \gamma)}{h^{2}} \cdot q(\alpha, \beta, \gamma) \cdot \cos ^{3} \gamma
$$

The r-table was developed as a description matrix of reflection properties in the terms of reduced luminance coefficients, given by

$$
r(\beta, \gamma)=q(\beta, \gamma) \cdot \cos ^{3} \gamma
$$

In Equation (2), $\alpha$ is standardized as $1^{\circ}$, since for car driving the observation angle in the range from $0.5^{\circ}$ to $2^{\circ}$ is of interest. In this range, the value of $q$ does not change significantly with $\alpha$.

The reflection table ( $r$-table) well describes the reflection properties of a road surface. However, it is not a direct indicator of general information of reflection properties and not convenient for 
comparing different surfaces. $Q_{0}, S_{1}$ and $S_{2}$ were then introduced to set a categorization label of surface reflection properties. $Q_{0}$ as formulated as (3), defines the general lightness level of the road surface, is also called the average luminance coefficient.

$$
Q_{0}=\frac{1}{\Omega_{0}} \int_{0}^{\Omega_{0}} q d \Omega
$$

where

$q \quad$ is the luminance coefficient;

$\Omega_{0} \quad$ is the solid angle, measured from the point on the surface, containing all those directions from which light is incident that are taken into account in the averaging process.

The specular factor $S_{1}$ is defined as ratio of a reduced luminance coefficient which is generally large for specular reflection $\left(\beta=0^{\circ}, \tan \gamma=2\right)$ to one which is generally large for diffuse reflection $\left(\beta=0^{\circ}, \tan \gamma=0\right)$.

$$
S_{1}=\frac{r(0,2)}{r(0,0)}
$$

Similarly specular factor $S_{2}$ is ratio of the average luminance coefficient to a reduced luminance coefficient at $\left(\beta=0^{\circ}, \tan \gamma=0\right)$.

$$
S_{2}=\frac{Q_{0}}{r(0,0)}
$$

As recommended by CIE, road surfaces are categorized by the specular factor $S_{1}$. For dry road surfaces three classes are in use, the R, N and C. For wet surfaces (CIE, 1979), class W is in use and the categorization is made according to a modified specular factor $S_{1}{ }^{\prime}$ formulated as equation (6) for $S_{1, \text { wet }}>1$.

$$
\log \frac{S_{1}{ }^{\prime}}{0.147}=\frac{\log \frac{S_{1, \text { Wet }}}{0.147}}{1-\frac{Q_{0, W e t}}{0.687}}
$$

where

$$
\begin{array}{ll}
S_{1, W e t} & \text { is } S_{1} \text { for the wet conditions; } \\
Q_{0, W e t} & \text { is } Q_{0} \text { for the wet conditions. }
\end{array}
$$

For $S_{1, w e t} \leq 1, S_{1}^{\prime}=S_{1, \text { Wet }}$.

\section{Model}

In this paper we come up with an idea that is borrowed from the popular data science nowadays. To do this, we build up a so-called 'knowledge base', which is constructed by reliable sources. Since each r-table consists of 580 real numbers, in our formulation each of the r-table is described by a vector $\boldsymbol{r}_{\mathrm{i}} \in \mathbf{R}^{580}$. Suppose we have $\mathrm{N}$ known $\mathrm{r}$-tables, then the knowledge base $\mathbf{R}$ is basically a matrix of size $580-$ by- $\mathrm{N}$ of the form

$$
\mathbf{R}=\left[\boldsymbol{r}_{\mathbf{1}} \boldsymbol{r}_{\mathbf{2}} \cdots \boldsymbol{r}_{N}\right]
$$

The Modelling task is to predict the full $r$-table according to a small number of observed values of the r-table, for a considered road surface. To insure the modelling precision, these observed values should represent the characteristics of the considered road surface. In our previous study on modelling ( $\mathrm{Li}, 2014$ ), we found that some of the points were of the most importance, of which their angle indices in the r-table were

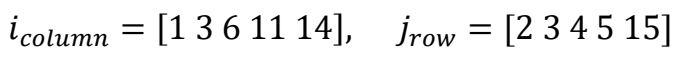


By using these 25 reduced luminance coefficients, the prediction errors were the smallest. So a measurement device based on model prediction method was built to measure the reduced luminance coefficients of these points. In this paper, we still use these 25 points.

First we reduce the dimension of the knowledge space. We define the projection operation $\mathbf{P}$ : $\mathbf{R}^{580} \rightarrow \mathbf{R}^{25}$, which projects each $\mathrm{r}$-table into a vector of 25 real numbers, i.e. taking values at the points with indices in Equation (8). Then a deflated knowledge base matrix $\widehat{\mathbf{R}}$ of size 25-by$\mathrm{N}$ is setup. To make a model prediction for a considered road surface, denote its 25 measured values as vector $\hat{\boldsymbol{r}}$, then we solve the following least square problem for $\boldsymbol{w}$

$$
\min _{W}\|\hat{\boldsymbol{r}}-\widehat{\mathbf{R}} \cdot \boldsymbol{w}\|_{2}
$$

where

$w$ is a vector with $\mathrm{N}$ weighting factors of the considered road surface for that knowledge base.

This problem has an explicit solution

$$
\boldsymbol{w}=\left(\widehat{\mathbf{R}}^{\mathrm{T}} \cdot \widehat{\mathbf{R}}\right)^{-1} \cdot \widehat{\mathbf{R}}^{\mathrm{T}} \cdot \widehat{\boldsymbol{r}}
$$

provided that the deflated knowledge base $\widehat{\mathbf{R}}$ has full column rank, so that the inverse part $\widehat{\mathbf{R}}^{\mathrm{T}}$. $\widehat{\mathbf{R}}$ is non-singular. Finally we can make the model prediction by

$$
\tilde{\boldsymbol{r}}=\mathbf{R} \cdot \boldsymbol{w}=\mathbf{R} \cdot\left(\widehat{\mathbf{R}}^{\mathrm{T}} \cdot \widehat{\mathbf{R}}\right)^{-1} \cdot \widehat{\mathbf{R}}^{\mathrm{T}} \cdot \widehat{\boldsymbol{r}}
$$

where

$\tilde{\boldsymbol{r}} \quad$ is the predicted $\mathrm{r}$-table vector with full 580 values;

$\mathbf{R} \quad$ is the knowledge base defined in Equation (7).

\section{Verification}

With the reliable r-tables we have at hand, we then perform some numerical experiments to verify the precision of our modelling method. First 287 verified r-tables of dry roads are used. We choose r-tables with even number indices to setup the knowledge base $\mathbf{R}$, and try to build up a model to predict the r-tables with odd number indices, assuming their reduced luminance coefficients with indices defined in Equation (8) have been measured but others have been not. After the predicted full r-table vector $\tilde{\boldsymbol{r}}$ of a road surface is obtained, the relative prediction errors on $Q_{0}, S_{1}, S_{2}$ and r-table are calculated and plotted in Figure 2, in which the relative error on $r$-table is defined as

$$
\operatorname{Err}(\|\boldsymbol{r}\|)=\frac{\|\tilde{r}-r\|_{2}}{\|r\|_{2}}
$$

where

$r \quad$ is the real full $r$-table vector of that road surface, obtained from the 287 r-table database. 


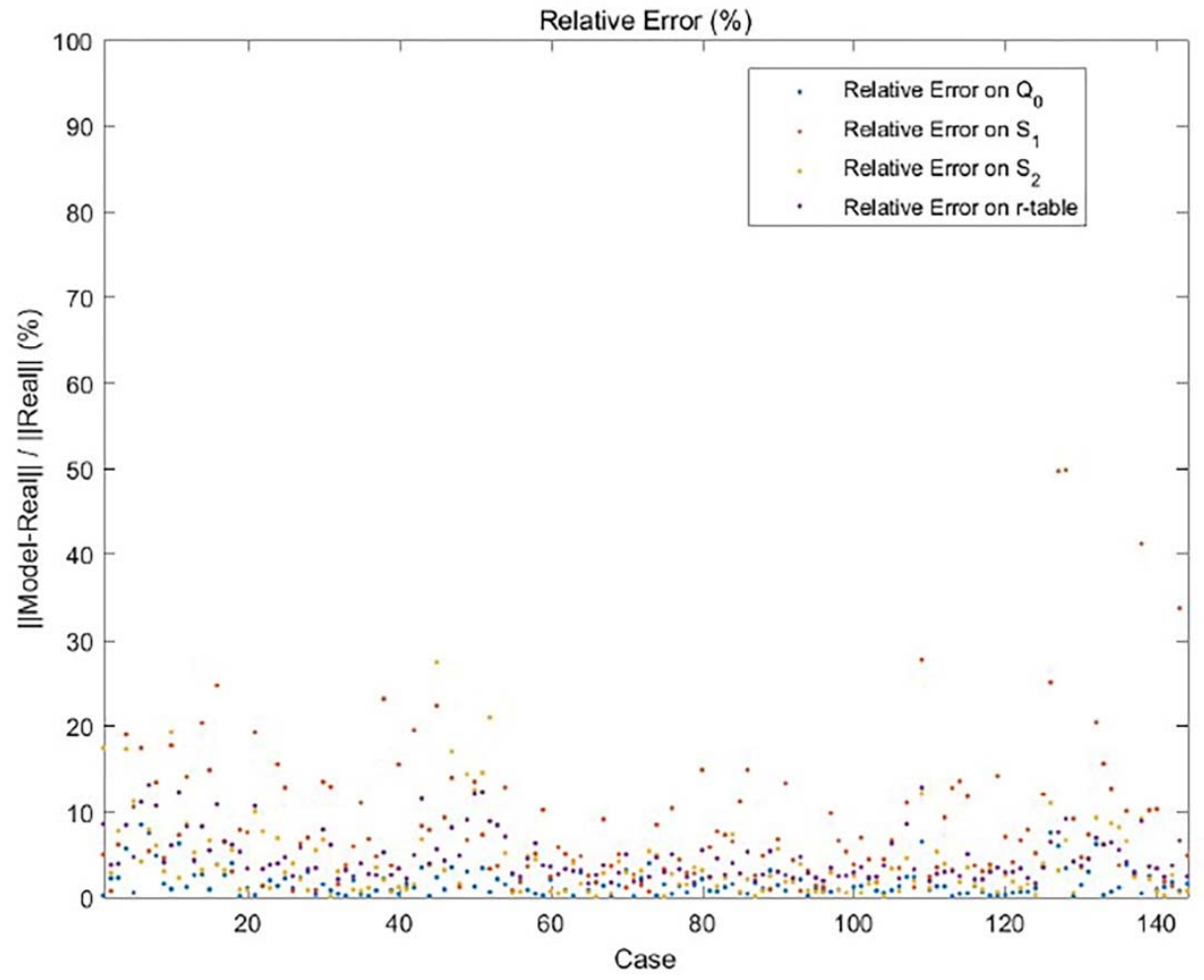

Figure 2 - Relative model prediction errors on $Q_{0}, S_{1}, S_{2}$ and r-table, using half of the $287 \mathrm{r}$ tables as database to predict the other half

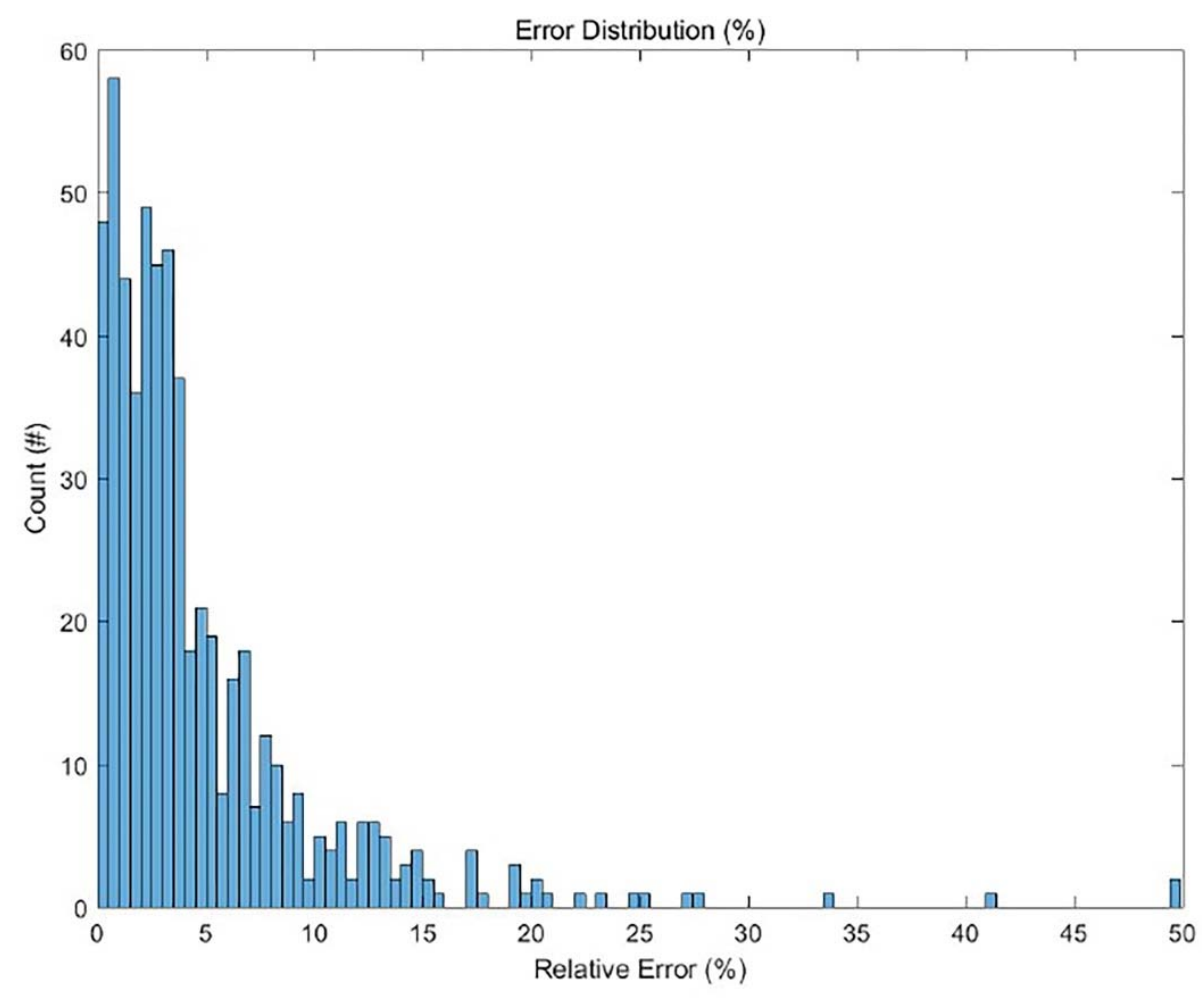

Figure 3 - Histogram of all the relative prediction errors in Figure 2

Figure 3 make a histogram of all the relative errors in Figure 2 . We find that $90 \%$ of the prediction errors are below $15 \%$, and a great number of them are distributed around $5 \%$. Only two worst cases are around $50 \%$. This is better than the results of all the four models. 


\section{Summary and outlook}

The database model proposed in this paper is adequate for a precise road light calculation, for the 287 r-tables of dry road surfaces tested in this paper. Our next step is to gather and verify more r-tables to expand our database, to make further verification for our model. Moreover, more r-tables covering road surfaces of different materials, service and weather conditions, especially wet road surfaces need to be modelled. Previous study showed that wet road surfaces were very difficult to model. Besides gathering verified wet r-tables (Frederiksen, 1976, Sorensen, 1974), we also setup a measurement system to obtain wet r-tables (Li, 2017, Wang, 2018), so that we can make predictions for wet road surfaces.

\section{Acknowledgement}

This work is supported by the National Key R\&D Program of China (Grant No. 2017YFB0403504).

\section{References}

Ahsen Erbay. Of the reflection properties of road surfaces. PhD dissertation, Technische University of Berlin, 1974.

Sorensen K., Nielsen B.. Road surfaces in traffic lighting. Report No. 9. The Danish Illuminating Engineering Laboratory, Lyngby, 1974.

E. Frederiksen and Kai Sorensen, Reflection classification of dry and wet road surfaces, Lighting Research and Technology, v 8, n 175, 1976.

CIE 047-1979, Road lighting for wet conditions.

CIE 66-1984, Road surfaces and lighting.

Gregory J. Ward. Measuring and modeling anisotropic reflection. Computer graphics, V 26, n 2, 1992.

N. Debergh and J.J. Embrechts. Mathematical modelling of reduced luminance coefficients for dry road surfaces. Lighting Research and Technology, v 40, 2008.

Li Wenyi, Zeng Xu. Modelling of road surface reflection property. Philips Technical Note PR-TN 2014/00447, 2014.

Li Wenyi, Zeng Xu, Zhu Xiaoyan, Wang Gang, Liu Muqing, Shen Haiping. Measurement system and method for reflection properties of wet road surfaces. CIE 2017 mid-term meeting, Jeju, 2017.

Wang Gang, Li Wenyi, Zeng Xu, Liu Muqing, Shen Haiping. Reflective characteristics of pavement on rainy days and its influence on road lighting quality. China Illuminating Engineering Journal, v 29, n 4, 2018. 